\title{
Adequate Energy Intake Prevents Low Bone Mass Under Exercise and Low Intake of Nutrients in Young Female Rats
}

\author{
Yuki Aikawa $^{1,2}$, Yuya Kakutani ${ }^{1,3}$, Umon Agata ${ }^{1,4}$, Satoshi Hattori ${ }^{1,5}$, Hitomi Ogata ${ }^{6}$, Ken Kiyono ${ }^{7}$, \\ Ikuko Ezawa $^{8}$, Naomi Omi ${ }^{9,}$, \\ ${ }^{1}$ Comprehensive Human Sciences, University of Tsukuba, Tsukuba, Japan \\ ${ }^{2}$ Department of Science of Living, Tsu City College, Tsu, Japan \\ ${ }^{3}$ Faculty of Health and Nutrition, Osaka Shoin Women's University, Higashi-Osaka, Japan \\ ${ }^{4}$ Department of Pharmaceutical and Medical Business Sciences, Nihon Pharmaceutical University, Ina-machi, Japan \\ ${ }^{5}$ Faculty of Sport Science, Waseda University, Tokorozawa, Japan \\ ${ }^{6}$ Graduate School of Integrated Arts and Sciences, Hiroshima University, Higashi-Hiroshima, Japan \\ ${ }^{7}$ Graduate School of Engineering Science, Osaka University, Toyonaka, Japan \\ ${ }^{8}$ Department of food and nutrition, Japan Women's University, Bunkyo-ku, Japan \\ ${ }^{9}$ Faculty of Health and Sport Sciences, University of Tsukuba, Tsukuba, Japan
}

\section{Email address:}

omi.naomi.gn@u.tsukuba.ac.jp (N. Omi)

${ }^{*}$ Corresponding author

\section{To cite this article:}

Yuki Aikawa, Yuya Kakutani, Umon Agata, Satoshi Hattori, Hitomi Ogata, Ken Kiyono, Ikuko Ezawa, Naomi Omi. Adequate Energy Intake Prevents Low Bone Mass Under Exercise and Low Intake of Nutrients in Young Female Rats. American Journal of Sports Science. Vol. 7, No. 3, 2019, pp. 127-135. doi: 10.11648/j.ajss.20190703.17

Received: August 14, 2019; Accepted: September 9, 2019; Published: September 19, 2019

\begin{abstract}
We examined the effects of adequate energy intake on bone strength and bone mass under exercise and low nutrient intake using a rat model of the female athlete triad (FAT). Seven-week-old female rats were divided into four groups: sedentary and ad libitum feeding group (SED), exercise and ad libitum feeding group (EX), exercise and $30 \%$ food restriction group (EX-FR), and exercise, $30 \%$ food restriction and adequate energy intake group (EX-FR + Ene). Excise groups were performed a voluntary running. The EX-FR + Ene group was fed glucose ad-libitum to adequate energy intake. The experiment lasted for 12 weeks. The energy availability, internal organ weight, bone size, bone strength, bone mass, and calcium absorption in the EX-FR group were significantly lower than those in the EX group. There were no significant differences in these parameters except bone strength in between the EX and EX-FR + Ene groups. The breaking energy in the EX-FR + Ene group was significantly lower than those in the EX group. Our results provide evidence that adequate energy intake is important for optimal bone growth in young female athletes.
\end{abstract}

Keywords: Female Athlete Triad, Food Restriction, Energy Supplementation, Bone Strength, Running Exercise

\section{Introduction}

Low bone mass, one of the components of the female athlete triad (FAT), is a serious problem for young female athletes [1]. Bone health is a crucial factor in an athlete's life and performance; if athletes do not have good bone strength, the risk of fracture increases [2]. The symptoms of FAT, such as low food intake, adversely affects bone growth in young athletes [1]. Furthermore, Low energy availability, corresponding to the amount of dietary energy remaining for other body functions after exercise, impairs bone health and affects menstrual functions in females $[1,3]$. Thus, FAT is a serious problem for young female athletes [1].

Optimizing energy availability is recommended to prevent low bone mass due to FAT [1]. Previous animal studies have reported that energy restriction by reducing carbohydrates alone in sedentary female rats resulted in bone loss, although other nutrients were not restricted [4, 5]. Moreover, in 
exercising female rats, Swift et al. reported that reducing all nutrients or restriction of energy by reducing carbohydrates alone resulted in bone loss; however, calcium (Ca) restriction did not result in bone loss [6]. These reports suggest that inadequate energy intake negatively affects bone health in sedentary and exercising women. However, it is unclear if adequate energy condition under low nutrient intake of prevents bone impairment in young female athletes. If energy availability strongly affects the bone, only optimal energy availability can induce optimal bone growth.

A rat model of FAT enabled us to examine whether only adequate energy conditions with a low intake of other nutrients prevents bone impairment. Dimarco et al. created an animal model of FAT in mature rats [7], and we used young female rat models with a combination of voluntary running exercise and $30 \%$ food restriction [8]. The animal model of FAT expresses the symptoms of FAT in humans, including low energy availability, estrous dysfunction, and low bone mass. Moreover, we previously shown that the interaction of voluntary running exercise and food restriction results in lower energy availability, bone strength, and bone mass than exercise or food restriction alone in young female rats [8]. This report suggests that preventing low energy availability is important for optimal bone growth. We hypothesized that adequate energy intake alone prevents bone impairment and FAT caused by food restriction even if various nutrient intakes are low.

The aim of the present study was to examine the effects of adequate energy intake on bone strength and bone mass under the condition of exercise and low nutrient intake using a rat model of FAT. In addition, we examined the preventive effects of Ca supplementation on the bone under exercise and low nutrient intake conditions. We previously reported that Ca supplementation has a positive effect on bone strength, but the effect was insufficient to prevent lower bone strength caused by inadequate food intake in a rat model of FAT [9]. We recently completed our analysis of the data on the effects of adequate energy intake on the bone in rat model. Therefore, in this report, we have included our previous data [9] along with the analysis of our new data from the adequate energy intake trials, and described the preventive effect of adequate energy intake on the impairment of bone strength and bone mass under exercise and low nutrient intake conditions.

\section{Materials and Methods}

\subsection{Experimental Design}

Female Sprague-Dawley rats $(\mathrm{n}=29,7$ weeks old) were randomly divided into following four experimental groups after a 1-week acclimatization period: sedentary and ad libitum feeding group (SED, $\mathrm{n}=7$ ), voluntary running exercise and ad libitum feeding group $(\mathrm{EX}, \mathrm{n}=7)$, voluntary running exercise and 30\% food restriction group (EX-FR, $n=$ 7 ), and voluntary running exercise, $30 \%$ food restriction and adequate energy intake group (EX-FR + Ene, $n=8)$. The experiment period was 12 weeks. The rats were purchased from CLEA Japan (Tokyo, Japan). We used 7 weeks old female rats as a model of adolescent girls. The elevation of serum estradiol level and vaginal opening occur in the age [10]. The experiment period is needed for low bone strength by interaction of running and food restriction.

The rats were fed normal diet containing $62.37 \%$ glucose monohydrate, $18.0 \%$ casein, $10.0 \%$ cottonseed oil, and others (crude $0.6 \% \mathrm{Ca}, 0.6 \% \mathrm{P}$ ) as previously described [8]. Food restriction groups were fed a $30 \%$ restricted diet containing $70 \%$ of the mean amount consumed in the previous week by the SED group. As a result, food restriction groups were fed the mean of $35 \%$ restricted diet compared to the EX group. Additionally, the EX-FR + Ene group was fed ad-libitum glucose to supply energy. We relied on a feeding instinct of rats for the method to supply the adequate energy because energy requirement of rats was unclear.

The SED group was individually housed in normal cages $(15 \times 25 \times 19.5 \mathrm{~cm})$, whereas the EX, EX-FR and EX-FR + Ene groups were individually housed with free access to voluntary running exercise on a wheel (wheel circumference, $1 \mathrm{~m}$; cage, $27 \times 35 \times 35 \mathrm{~cm}$ ). We used the voluntary running scheme to assess the changing daily running distance. The room was maintained at $22 \pm 1{ }^{\circ} \mathrm{C}$ under a constant $12: 12 \mathrm{~h}$ light-dark cycle (light 8:00 to 20:00). Animal care and experimental procedures were approved by the Animal Experimental Committee of the University of Tsukuba (approval number: 11-377).

\subsection{Daily Data Collection and Specimen Harvesting}

The body weight and dietary intake were measured every second day, and the running distance was measured every day. On the day prior to the dissection, all rats were made to fast for $12 \mathrm{~h}$. Whole blood samples were collected from the abdominal aorta using syringes under diethyl ether anesthesia. Serum samples were separated by centrifugation at $2500 \mathrm{rpm}$ for 20 $\min$ at $4^{\circ} \mathrm{C}$. The serum was frozen at $-80^{\circ} \mathrm{C}$ for the determination of bone metabolic markers. The abdominal fat, plantaris muscle, soleus muscle, uterus, adrenal gland, thymus, femur, tibia, and lumbar spine were collected from each rat after death. Femurs were collected, adhering connective tissues were removed, wet femur weight and size were measured, and bone strength was immediately measured. Subsequently, the femur was dried at $100{ }^{\circ} \mathrm{C}$ for $24 \mathrm{~h}$ in an electric furnace and their dry weight was measured. Next, dried femurs were burned to ash at $600{ }^{\circ} \mathrm{C}$ for $15 \mathrm{~h}$, and the ash weight was measured. The tibia and lumbar spine were stored in $70 \%$ ethanol after being harvested and cleaned of soft tissue for the measurement of the bone area, bone mineral content (BMC), and BMD. The animals were placed in individual metabolic cages $(24 \times 20 \times 18 \mathrm{~cm})$ on the 80 th and 81 st day just before the end of the experimental period. Urine and fecal matter were collected over two $24-\mathrm{h}$ periods. Urine was collected under acidic conditions using $2 \mathrm{~mL} 2 \mathrm{~N}$ hydrochloric acid. The urine was centrifuged at $2500 \mathrm{rpm}$ for $15 \mathrm{~min}$ to eliminate refuse. 


\subsection{Calculation of Energy Availability}

The energy intake, exercise-induced energy expenditure, and energy availability were calculated as previously described $[8,9]$. Briefly, energy intake was calculated by multiplying the amount of normal diet intake $(3.73 \mathrm{kcal} / \mathrm{g})$ and glucose intake $(3.36 \mathrm{kcal} / \mathrm{g})$. Exercise-induced energy expenditure due to daily wheel-running was calculated as 5.0 $\mathrm{kcal} / \mathrm{kg}$ body weight times the $\mathrm{km}$ run [11], as in a previous study (exercise-induced energy expenditure $=$ wheel-running distance $\times$ body weight $\times 5.0 \mathrm{kcal} / \mathrm{kg}$ body weight $/ \mathrm{km}$ ) [7]. Energy availability was calculated as energy intake minus exercise energy expenditure [7].

\subsection{Evaluation of Estrous Cycle by Spectral Analysis of the Running Distance}

This analysis was performed to determine whether the running distance increased every 4 or 5 days to assess the estrous cycle using previously described methods [8]. If the running distance cyclically increases every 4 or 5 days, the proportion of the power spectrum of the running distance from 0.2 to $0.3 \mathrm{~Hz}$ in the total power spectrum will be high. In contrast, a minimal wheel-running fluctuation has been reported in anestrous female rats [7]. Therefore, if the female rats are anestrous, the proportion of the power spectrum of the running distance from 0.2 to $0.3 \mathrm{~Hz}$ in the total power spectrum will be low.

\subsection{Measurement of Bone Strength Using Three-Point Bending Test}

The strength of the femoral mid-shaft was assessed using a three-point bending test (DYN-1255, IIO DENKI, Tokyo, Japan) as previously described (distance between the fulcrums, $1 \mathrm{~cm}$; plunger speed, $100 \mathrm{~mm} / \mathrm{min}$; full scale, $50 \mathrm{~kg}$; and chart speed, $120 \mathrm{~cm} / \mathrm{min}$ ) [12]. Breaking force referred to the loading weight (gravitational acceleration) required for bone breaking. Breaking energy referred to the workload that resulted in the breaking of the bone.

\subsection{Measurement of BMC, Bone Area and BMD Using Dual-Energy X-ray Absorptiometry}

The BMC, bone area and BMD values of the tibia and L3-L6 lumbar spine were measured by dual energy X-ray absorptiometry (DXA; Aloka, DCS-600R, Tokyo, Japan) as previously described [13].

\subsection{Ca Balance Study}

Ca balance study was measured as previously described [13]. All feces were burned to ash at $600^{\circ} \mathrm{C}$ for $15 \mathrm{~h}$, and the fecal matter was dissolved in $1 \mathrm{~N}$ nitric acid. $\mathrm{Ca}$ content in urine and feces was measured using inductively coupled plasma atomic emission spectroscopy (ICAP-AES; $575 \mathrm{~V}$, Nippon Jarrell-Ash). Ca absorption and accumulation were calculated using $\mathrm{Ca}$ intake and fecal and urinary excretion of $\mathrm{Ca}$. Amount of $\mathrm{Ca}$ absorption $(\mathrm{mg} / \mathrm{day})=\mathrm{Ca}$ intake minus fecal $\mathrm{Ca}$ excretion. Rate of $\mathrm{Ca}$ absorption $(\%)=$ amount of $\mathrm{Ca}$ absorption divided by $\mathrm{Ca}$ intake multiplied by 100 . Amount of $\mathrm{Ca}$ accumulation $(\mathrm{mg} /$ day $)=$ amount of $\mathrm{Ca}$ absorption minus urine $\mathrm{Ca}$ excretion. Rate of $\mathrm{Ca}$ accumulation (\%) = amount of $\mathrm{Ca}$ accumulation divided by $\mathrm{Ca}$ intake multiplied by 100 .

\subsection{Statistical Analysis}

All data were expressed as mean \pm standard error (SE). Statistical analysis was conducted using one-way analysis of variance (ANOVA). In any analysis, if significant difference was observed, the variables in EX, EX-FR, and EX-FR + Ene groups were analyzed using the Tukey's post-hoc comparison tests. SED group data were not included in ANOVA. Unpaired $t$ tests were used to compare results for SED and EX groups to assess the effect of exercise. The significance level was set at $p<0.05$. All statistical analysis was performed using SPSS Statistical Packages (Ver. 19.0; SPSS Inc., Chicago, USA).

\section{Results}

\subsection{Food Intake, Running Distance, and Energy Availability}

Table 1 presents the food intake and energy availability. The normal diet intake in the EX-FR + Ene group was significantly lower than that in the EX group. There were no significant difference between the EX-FR and EX-FR + Ene groups. The total food intake, including glucose intake, in the EX-FR + Ene group was significantly higher than that in the EX and EX-FR groups. The Ca intake in the EX-FR + Ene group was significantly lower than that in the EX group. The energy intake in the EX-FR + Ene group was significantly higher than that in the EX group. There were no significant differences in the wheel running distance and exercise-induced energy expenditure in each group. There were no significant differences in the percentage of the power spectrum of the running distance and energy availability between the EX and EX-FR + Ene groups.

Table 1. Food intake, running distance, and energy availability.

\begin{tabular}{lllll}
\hline & SED & EX & EX-FR & EX-FR+Ene \\
\hline Normal food intake (g/day) & $17.8 \pm 0.6^{*}$ & $19.6 \pm 0.3^{\mathrm{a}}$ & $12.6 \pm 0.0^{\mathrm{b}}$ & $12.6^{\mathrm{b}} \pm 0.0^{\mathrm{b}}$ \\
Glucose intake (g/day) & - & - & - & $9.0 \pm 0.5$ \\
Total food intake (g/day) & $17.8 \pm 0.6^{*}$ & $19.6 \pm 0.3^{\mathrm{b}}$ & $12.6 \pm 0.0^{\mathrm{c}}$ & $21.6 \pm 0.5^{\mathrm{a}}$ \\
Ca intake (mg/day) $_{\text {Energy intake }(\mathrm{kcal} / \text { day) }}$ & $107 \pm 3^{*}$ & $118 \pm 2^{\mathrm{b}}$ & $76 \pm 0^{\mathrm{c}}$ & $76 \pm 0^{\mathrm{c}}$ \\
Wheel running distance (km/day) & $66.3 \pm 2.1^{*}$ & $73.3 \pm 1.2^{\mathrm{b}}$ & $47.3 \pm 0.1^{\mathrm{c}}$ & $77.2^{\mathrm{a}} \pm 1.6^{\mathrm{a}}$ \\
\hline
\end{tabular}




\begin{tabular}{lllll}
\hline & SED & EX & EX-FR & EX-FR+Ene \\
\hline Percentage of the power spectrum of the running distance $^{2}$ (Ratio) & - & $0.44 \pm 0.04^{\mathrm{a}}$ & $0.17 \pm 0.04^{\mathrm{b}}$ & $0.42 \pm 0.06^{\mathrm{a}}$ \\
Exercise-induced energy expenditure $^{3}$ (kcal/day) & - & $11.4 \pm 1.6$ & $10.7 \pm 0.5$ & $12.0 \pm 1.4$ \\
Energy availability $^{4}(\mathrm{kcal} /$ day) & $66.3 \pm 2.1$ & $61.9 \pm 1.4^{\mathrm{a}}$ & $36.5 \pm 0.5^{\mathrm{b}}$ & $65.1 \pm 2.2^{\mathrm{a}}$ \\
\hline
\end{tabular}

SED: sedentary group. EX: exercise group. EX+FR: exercise + food restriction group. EX-FR + Ene: exercise + food restriction + adequate energy intake group. Values are expressed as means \pm SE. The values are expressed as the means of average of the entire experimental period. Unpaired t tests were used to compare results for SED group and EX group to assess the effect of exercise. * $\mathrm{p}<0.05$ for vs. EX group.

Data in EX, EX-FR, and EX-FR + Ene groups were analyzed by the Tukey's post-hoc comparison test. Means with unlike alphabet are significantly different.

${ }^{1}$ Energy intake was calculated by multiplying the amount of daily food intake in grams by the energy content of the food (normal diet, $3.73 \mathrm{kcal} / \mathrm{g}$; glucose diet, $3.36 \mathrm{kcal} / \mathrm{g})$.

${ }^{2}$ To quantify the periodic component at approximately 4 or 5 days in the temporal profile of the running distance, we estimated the power in the frequency band from 0.2 to $0.3 \mathrm{~Hz}$ using spectral analysis of the detrended time series. Moreover, to exclude the inter-individual difference in the total power affecting the local power, we also computed the proportion of the power spectrum of the running distance from 0.2 to $0.3 \mathrm{~Hz}$ in the total power spectrum [8].

${ }^{3}$ Exercise-induced energy expenditure from daily wheel running was calculated as $5.0 \mathrm{kcal} / \mathrm{kg}$ body weight times kilometers run [11].

${ }^{4}$ Energy availability was calculated as energy intake minus exercise energy expenditure.

\subsection{Body Weight and Internal Organ Weight}

The body weight and internal organ weight at dissection are presented in Table 2. There were no significant differences in the final body weight, abdominal fat, plantaris muscle, soleus muscle, uterus, adrenal gland, and thymus weight between the EX and EX-FR + Ene groups.

Table 2. Body weight and internal organ weight.

\begin{tabular}{lllll}
\hline & SED & EX & EX-FR & EX-FR+Ene \\
\hline Initial body weight $(\mathrm{g})$ & $208 \pm 3$ & $208 \pm 3$ & $210 \pm 4$ & $209 \pm 3$ \\
Final body weight $(\mathrm{g})$ & $340 \pm 15^{*}$ & $295 \pm 5^{\mathrm{a}}$ & $194 \pm 4^{\mathrm{b}}$ & $308 \pm 12^{\mathrm{a}}$ \\
Abdominal fat weight $(\mathrm{g})$ & $26.0 \pm 5.3^{*}$ & $12.4 \pm 1.7^{\mathrm{a}}$ & $1.6 \pm 0.2^{\mathrm{b}}$ & $16.3 \pm 2.1^{\mathrm{a}}$ \\
Plantaris muscle weight $(\mathrm{g})$ & $0.34 \pm 0.02$ & $0.34 \pm 0.01^{\mathrm{a}}$ & $0.25 \pm 0.01^{\mathrm{b}}$ & $0.34 \pm 0.01^{\mathrm{a}}$ \\
Soleus muscle weight $(\mathrm{g})$ & $0.111 \pm 0.005^{*}$ & $0.138 \pm 0.005^{\mathrm{a}}$ & $0.095 \pm 0.006^{\mathrm{b}}$ & $0.129 \pm 0.005^{\mathrm{a}}$ \\
Uterus weight $(\mathrm{g})$ & $0.53 \pm 0.03$ & $0.62 \pm 0.04^{\mathrm{a}}$ & $0.32 \pm 0.07^{\mathrm{b}}$ & $0.59 \pm 0.06^{\mathrm{a}}$ \\
Adrenal gland weight $(\mathrm{g})$ & $0.037 \pm 0.001$ & $0.043 \pm 0.003^{\mathrm{a}}$ & $0.033 \pm 0.002^{\mathrm{b}}$ & $0.041 \pm 0.003^{\mathrm{ab}}$ \\
Thymus weight $(\mathrm{g})$ & $0.29 \pm 0.03$ & $0.23 \pm 0.08^{\mathrm{ab}}$ & $0.18 \pm 0.05^{\mathrm{b}}$ & $0.30 \pm 0.04^{\mathrm{a}}$ \\
\hline
\end{tabular}

SED: sedentary group. EX: exercise group. EX+FR: exercise + food restriction group. EX-FR + Ene: exercise + food restriction + adequate energy intake group. Values are expressed as means $\pm \mathrm{SE}$. The values are expressed as the means of average of the entire experimental period. Unpaired tests were used to compare results for SED group and EX group to assess the effect of exercise. *p $<0.05$ for vs. EX group.

Data in EX, EX-FR, and EX-FR + Ene groups were analyzed by the Tukey's post-hoc comparison test. Means with unlike alphabet are significantly different.

\subsection{Bone Strength, BMD, Bone Weight, Bone Size, BMC, and Bone Area}

The breaking force and breaking energy of the femur are presented in Figure 1. The breaking energy as well as the breaking force of the femur in the EX group were significantly higher than those in the SED group. The breaking force and

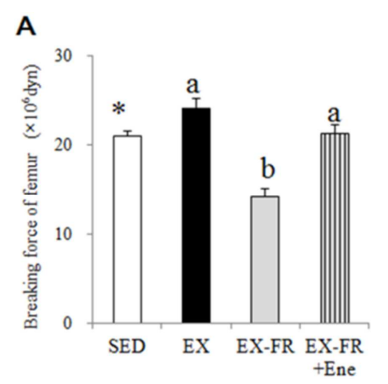

energy in in the EX-FR + Ene group was significantly higher than those in the EX-FR group. Meanwhile, the breaking energy of the femur in the EX-FR + Ene group was significantly lower than that in the EX group. There was no significant difference in the breaking force of the femur between the EX and EX-FR + Ene groups.

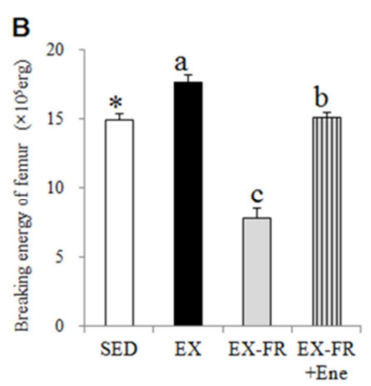

Figure 1. Breaking force and breaking energy of the femur.

(A): Breaking force of femur. (B): Breaking energy of femur.

SED: sedentary group. EX: exercise group. EX+FR: exercise + food restriction group. EX-FR + Ene: exercise + food restriction + adequate energy intake group. Values are expressed as means $\pm \mathrm{SE}$. The values are expressed as the means of average of the entire experimental period. Unpaired tests were used to compare results for SED group and EX group to assess the effect of exercise. * $<<0.05$ for vs. EX group.

Data in EX, EX-FR, and EX-FR + Ene groups were analyzed by the Tukey's post-hoc comparison test. Means with unlike alphabet are significantly different. The BMD of the lumbar spine, total tibia, proximal metaphysis tibia, and diaphysis tibia are presented in Figure 2. There were no significant differences in these parameters between the EX, and EX-FR + Ene groups. 

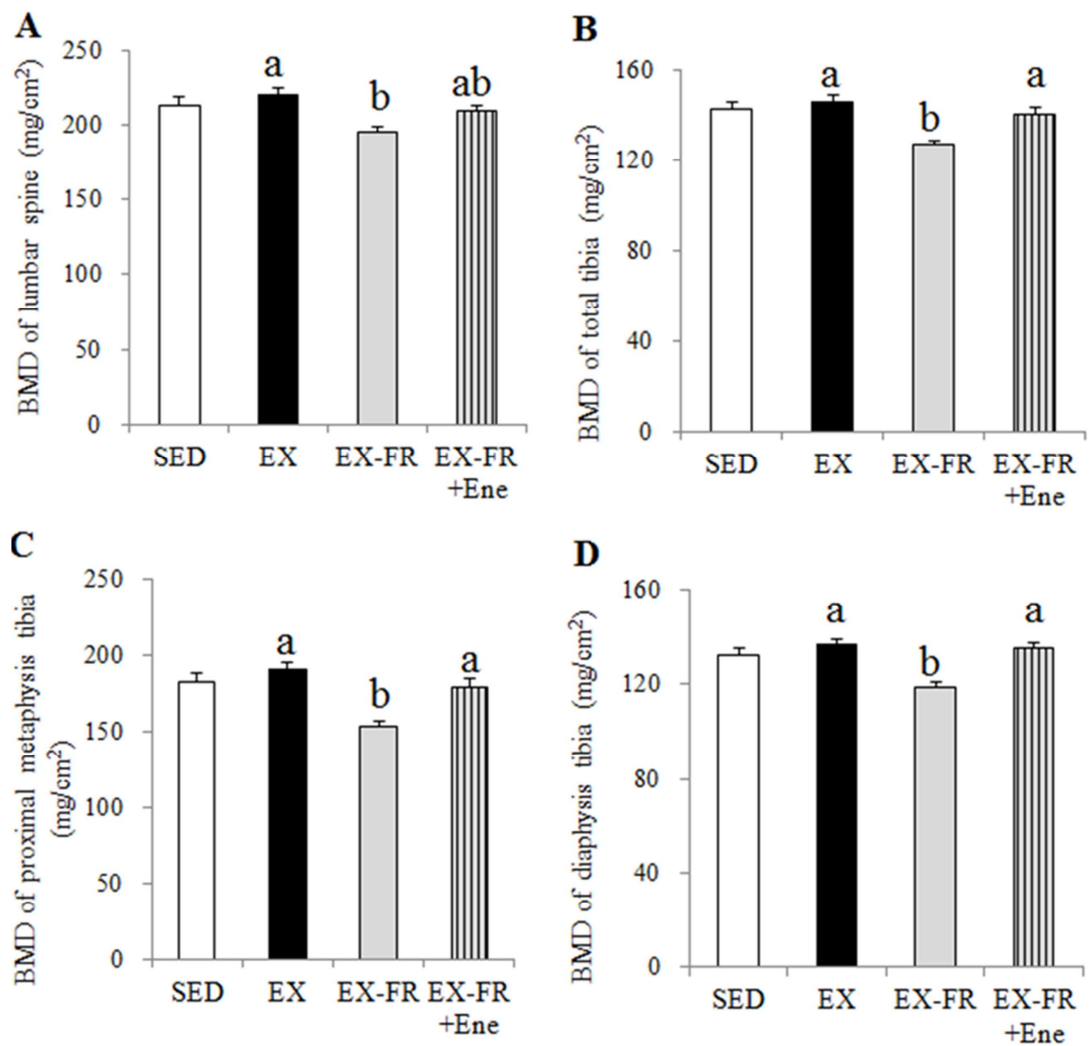

Figure 2. BMC, bone area, and BMD of the lumbar spine and tibia.

(A): Bone area of the lumbar spine. (B): BMD of the total tibia. (C): BMD of proximal metaphysis tibia. (D): BMD of diaphysis tibia.

SED: sedentary group. EX: exercise group. EX+FR: exercise + food restriction group. EX-FR + Ene: exercise + food restriction + adequate energy intake group. Values are expressed as means \pm SE. The values are expressed as the means of average of the entire experimental period. Unpaired tests were used to compare results for SED group and EX group to assess the effect of exercise. *p $<0.05$ for vs. EX group.

Data in EX, EX-FR, and EX-FR + Ene groups were analyzed by the Tukey's post-hoc comparison test. Means with unlike alphabet are significantly different. The weight of femur, size of femur, BMC of lumbar and tibia, and bone area of lumbar and tibia are presented in Table 3 . There were no significant differences in these parameters between the EX and EX-FR + Ene groups.

Table 3. Bone weight and size.

\begin{tabular}{lllll}
\hline & SED & EX & EX-FR & EX-FR+Ene \\
\hline Wet weight of femur (g) & $0.95 \pm 0.02$ & $0.92 \pm 0.01^{\mathrm{a}}$ & $0.74 \pm 0.02^{\mathrm{b}}$ & $0.89 \pm 0.02^{\mathrm{a}}$ \\
Dry weight of femur (g) & $0.61 \pm 0.01$ & $0.60 \pm 0.01^{\mathrm{a}}$ & $0.46 \pm 0.01^{\mathrm{b}}$ & $0.59 \pm 0.01^{\mathrm{a}}$ \\
Ash weight of femur (g) & $0.43 \pm 0.01$ & $0.42 \pm 0.01^{\mathrm{a}}$ & $0.30 \pm 0.01^{\mathrm{b}}$ & $0.40 \pm 0.01^{\mathrm{a}}$ \\
Length of femur (cm) & $3.63 \pm 0.03$ & $3.59 \pm 0.01^{\mathrm{a}}$ & $3.36 \pm 0.02^{\mathrm{b}}$ & $3.59 \pm 0.02^{\mathrm{a}}$ \\
Major axis of femur (cm) & $0.411 \pm 0.007$ & $0.396 \pm 0.006$ & $0.376 \pm 0.007$ & $0.396 \pm 0.007$ \\
Minor axis of femur (cm) & $0.332 \pm 0.004$ & $0.338 \pm 0.005^{\mathrm{a}}$ & $0.316 \pm 0.003^{\mathrm{b}}$ & $0.329 \pm 0.004^{\mathrm{ab}}$ \\
BMC of lumbar (m) & $558 \pm 26$ & $545 \pm 8.9^{\mathrm{a}}$ & $378 \pm 13^{\mathrm{b}}$ & $529 \pm 17^{\mathrm{a}}$ \\
Bone area of lumbar (cm $\left.{ }^{2}\right)$ & $2.61 \pm 0.07$ & $2.48 \pm 0.03^{\mathrm{a}}$ & $1.94 \pm 0.04^{\mathrm{b}}$ & $2.53 \pm 0.11^{\mathrm{a}}$ \\
BMC of total tibia (mg) & $284 \pm 11$ & $309 \pm 7^{\mathrm{a}}$ & $225 \pm 6^{\mathrm{b}}$ & $302 \pm 6^{\mathrm{a}}$ \\
Bone area of total tibia (cm $\left.{ }^{2}\right)$ & $1.97 \pm 0.05^{*}$ & $2.15 \pm 0.02^{\mathrm{a}}$ & $1.79 \pm 0.02^{\mathrm{b}}$ & $2.16 \pm 0.04^{\mathrm{a}}$ \\
BMC of proximal metaphysis tibia $(\mathrm{mg})$ & $92 \pm 4$ & $101 \pm 3^{\mathrm{a}}$ & $65 \pm 3^{\mathrm{b}}$ & $93 \pm 3^{\mathrm{a}}$ \\
Bone area of proximal metaphysis tibia $\left(\mathrm{cm}^{2}\right)$ & $0.50 \pm 0.02$ & $0.53 \pm 0.01^{\mathrm{a}}$ & $0.43 \pm 0.02^{\mathrm{b}}$ & $0.52 \pm 0.02^{\mathrm{a}}$ \\
BMC of diaphysis tibia (mg) & $108 \pm 4^{*}$ & $121 \pm 2^{\mathrm{a}}$ & $91 \pm 2^{\mathrm{b}}$ & $117 \pm 3^{\mathrm{a}}$ \\
Bone area of diaphysis tibia $\left(\mathrm{cm}^{2}\right)$ & $0.80 \pm 0.01^{*}$ & $0.90 \pm 0.02^{\mathrm{a}}$ & $0.77 \pm 0.01^{\mathrm{b}}$ & $0.87 \pm 0.02^{\mathrm{a}}$ \\
\hline
\end{tabular}

SED: sedentary group. EX: exercise group. EX+FR: exercise + food restriction group. EX-FR + Ene: exercise + food restriction + adequate energy intake group. Values are expressed as means \pm SE. The values are expressed as the means of average of the entire experimental period. Unpaired t tests were used to compare results for SED group and EX group to assess the effect of exercise. ${ }^{*} \mathrm{p}<0.05$ for vs. EX group.

Data in EX, EX-FR, and EX-FR + Ene groups were analyzed by the Tukey's post-hoc comparison test. Means with unlike alphabet are significantly different.

\subsection{Ca Balance Study}

Figure 3 presents the results of Ca balance study. The amount of $\mathrm{Ca}$ absorption and accumulation in the EX-FR group were significantly lower than those in the EX group, and the amount of Ca absorption in the EX-FR + Ene group was significantly lower than that in the EX group. There were no significant differences in other parameters between the EX and EX-FR + Ene groups. 
A

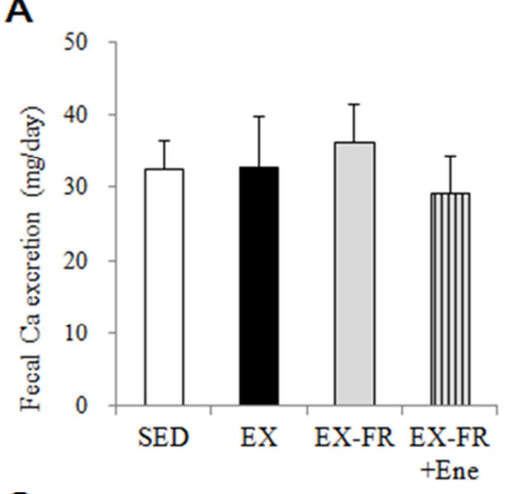

C

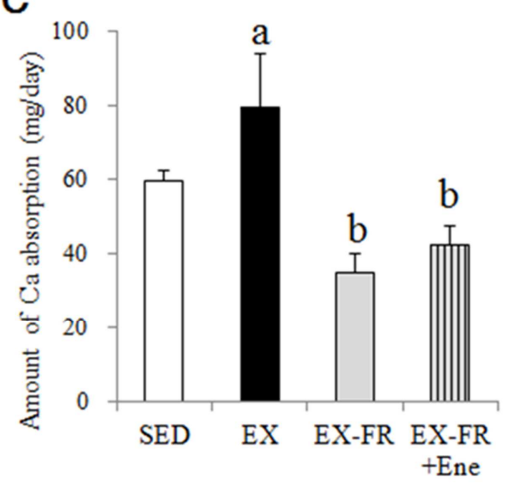

E

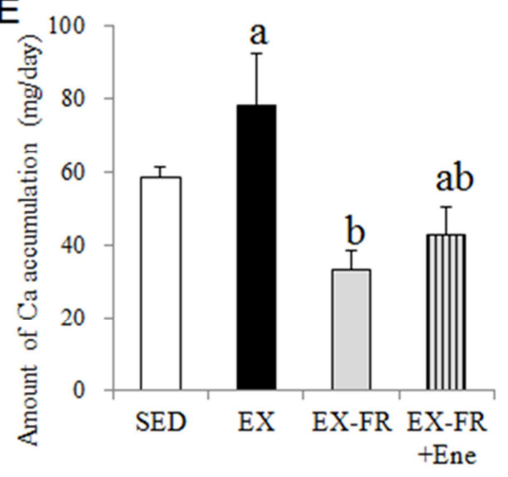

B

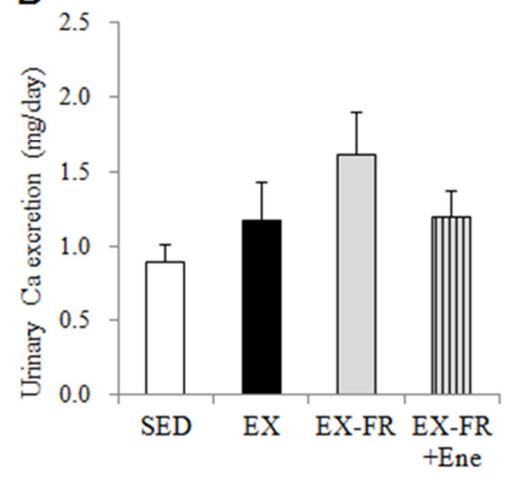

D
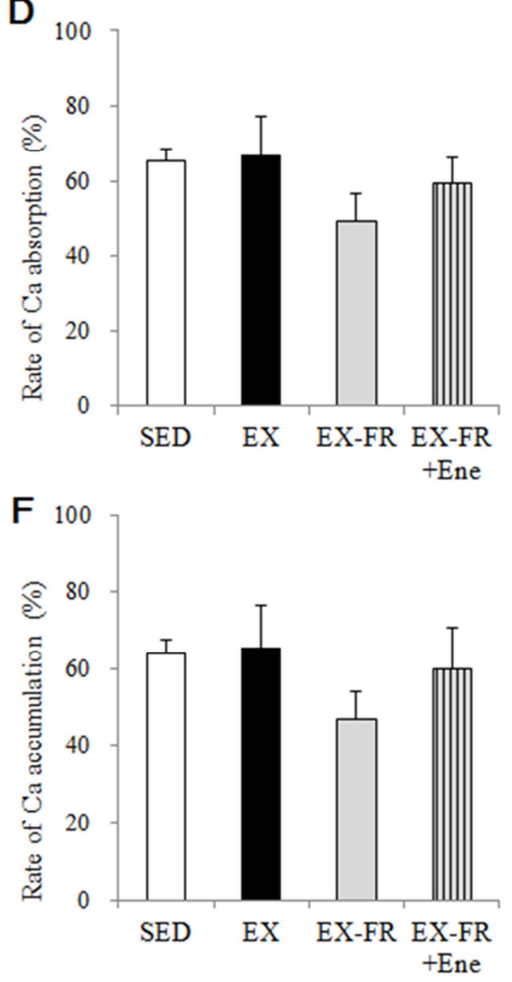

Figure 3. Ca balance study.

(A): Fecal Ca excretion. (B): Urinary Ca excretion. (C): Amount of Ca absorption. (D): Rate of Ca absorption. (E): Amount of Ca accumulation. (F): Rate of Ca accumulation.

SED: sedentary group. EX: exercise group. EX+FR: exercise + food restriction group. EX-FR + Ene: exercise + food restriction + adequate energy intake group. Values are expressed as means $\pm \mathrm{SE}$. The values are expressed as the means of average of the entire experimental period. Unpaired t tests were used to compare results for SED group and EX group to assess the effect of exercise. ${ }^{*} \mathrm{p}<0.05$ for vs. EX group.

Data in EX, EX-FR, and EX-FR + Ene groups were analyzed by the Tukey's post-hoc comparison test. Means with unlike alphabet are significantly different.

\section{Discussion}

The aim of the present study was to examine the effects of adequate energy intake on bone strength and bone mass under exercise and low nutrient intake conditions using a rat model of FAT. We reported the results of SED, EX, and EX-FR groups in our previous study [9]. The results of the present study demonstrate that adequate energy intake prevented lower energy availability, estrous dysfunction, smaller bone size, smaller bone area, lower BMC, and lower BMD in young female rats under exercise and low nutrient intake. However, lower bone strength was not prevented by adequate energy intake alone.
In the present study, we examined the effects of adequate energy intake on bone using only glucose supplementation. Our previous study reported that voluntary running exercise resulted in higher bone strength, and the interaction of voluntary wheel running and food restriction resulted in lower energy availability, internal organ weight, bone strength, and bone mass compared with exercise or food restriction alone in young female rats [8]. These results suggest that low energy availability in combination with voluntary wheel running and food restriction resulted in low bone strength and bone mass and was associated with menstrual dysfunction, as in FAT. In contrast, it is possible that a low intake of other nutrients rather than low energy availability affected the bone in the 
previous study [8]. Therefore, in the present study, we examined the effects of adequate energy intake on bone using only glucose supplementation as the main source of energy along with low intake levels of other nutrients in the rat model. We used glucose as the source of energy is because it is the main component of our normal diet and has less potential to affect the bone due to excess intake than other major nutrients, such as proteins or fats [15].

The present study shows that normal energy availability is a key factor for normal growth. Under exercise conditions, energy intake, energy availability, and body weight were not significantly different between ad libitum intake and food restriction with adequate energy intake (Tables 1 and 2). Despite the low intake of nutrients except energy, the inhibition of body weight and muscle weight was not observed in EX-FR + Ene group. These results are similar to those of our study that looked at providing low protein diets (10\%) to treadmill running young male rats [16]. Energy availability is defined as the energy intake minus exercise energy expenditure. Low energy availability suppresses various physiological functions, including cellular maintenance and growth [3], thus decreasing total energy expenditure [17]. The body weight results of the present study as well as our previous study suggest that it is more important to provide adequate energy to young rats for optimal growth rather than other nutrients. Furthermore, the present study demonstrated that low nutrient intake, except energy, did not lead to any reproductive dysfunction, as observed from the results of uterus weight and the percentage of the power spectrum of the running distance. Estrogen deficiency induces low uterus weight [18]. In case of estrous cycle disorder, the running cycle would have disappeared [7, 19] and the percentage of the power spectrum of the running distance would be low. Thus, we assumed that adequate energy intake could also prevent reproductive dysfunction in rat model. These results suggest that normal energy availability is a key factor in maintaining normal reproductive functions in young female rats engaged in voluntary wheel running.

Adequate energy intake alone caused higher bone strength compared with low energy intake under the conditions of voluntary wheel running and food restriction; however, it caused lower bone strength compared with wheel running and ad libitum food intake in young female rats (Figure 1). To the best of our knowledge, few studies have reported the influence of low nutrient intake, except energy, on bone strength. Our results show that adequate energy intake could not completely prevent the lower bone strength in the rat model.

BMD is frequently used as a proxy measure of bone strength and accounts for approximately $70 \%$ of bone strength [20]; therefore, it is the main factor in determining bone strength. In the present study, adequate energy intake prevented lower BMD in young female rats with voluntary wheel running and food restriction (Figure 2). In the previous study, our group reported that providing the same amount of food intake as that provided under sedentary conditions did not induce higher BMD in female rats under wheel running conditions; however, glucose supplementation resulted in higher BMD in female rats under similar conditions [21]. In other previous studies, $40 \%$ energy restriction by reducing only carbohydrate resulted in low BMD although other nutrients were not restricted in mature female rats $[4,5]$. The present and previous results suggest that energy availability has more influence on BMD than the intake of other nutrients. In the previous study, the results of BMC and bone area were similar to BMD; however, in the present study, bone strength results did not resemble $\mathrm{BMD}, \mathrm{BMC}$, and bone size results. Ackerman et al. reported no significant differences in BMD of the femoral neck between 0 fracture and $\geq 1$ fracture groups in adolescent amenorrheic athletes [22]. There might be some discrepancies between bone strength and BMD. Pando et al. reported that food restriction impaired bone quality, such as cortical area and trabecular number [23]. Our results and Pando et al.'s report suggest that changes in bone quality caused the impairment in bone strength by low nutrient intake, except energy, in young female rats under exercise conditions.

In young female rats, voluntary wheel running had no effect on bone length, and food restriction resulted in smaller bone length with voluntary wheel running conditions, and adequate energy intake prevented smaller bone length with voluntary wheel running and food restriction (Table 3). These results suggest that energy availability has a greater influence on bone size than other nutrients.

We hypothesized that voluntary wheel running, food restriction, and energy intake affect $\mathrm{Ca}$ absorption because energy restriction is reported to reduce fractional $\mathrm{Ca}$ absorption [24], and low $\mathrm{Ca}$ intake induces high $\mathrm{Ca}$ absorption rates $[25,26]$ in sedentary rats; therefore, the Ca balance was tested. In the present study, food restriction caused lower amount of $\mathrm{Ca}$ absorption and accumulation in young female rats with voluntary wheel running. Moreover, adequate energy intake had no effect on lower amount of $\mathrm{Ca}$ absorption but had a positive effect on lower amount of $\mathrm{Ca}$ accumulation in a rat model of FAT (Figure 3). These results suggest that $\mathrm{Ca}$ absorption is affected by $\mathrm{Ca}$ intake above the energy intake under exercise condition, and energy intake is one of the factors affecting $\mathrm{Ca}$ accumulation.

In the present study, under voluntary wheel running, the amount of $\mathrm{Ca}$ absorption and accumulation with low nutrient intake, except energy, were lower than those with ad libitum feeding, but the results of BMC and ash weight of the femur were not significantly different. Therefore, it is possible that adequate energy intake increases the ability of absorption of $\mathrm{Ca}$ from internal tissues to the bone in the rat.

We speculated that adequate energy intake alone prevented lower BMD by preventing abnormal hormone secretion, in the rat model of FAT, even under with the conditions of voluntary wheel running and food restriction. Low energy availability induces the suppression of physiological systems [3], including the secretion of hormones such as estradiol and IGF-1, affecting bone turnover and promoting bone strength [27]. In the present study, we did not measure these hormones; however, from the results of uterus weight and running cycle, 
we deducted that adequate energy intake alone also prevented low estradiol in the rat model. Further investigation into the alteration of hormone secretion and mechanisms by which energy intake prevents lower BMD in the rat model of FAT, even under conditions of voluntary wheel running and food restriction, are necessary.

This study has the following limitation. We used the method of ad libitum glucose feeding for adequate energy intake, which may also affect bone through the high intake of glucose, not energy alone.

\section{Conclusion}

Adequate energy intake could not completely prevent lower bone strength but did prevent lower body weight, muscle weight, bone size, bone area, BMC, and BMD with interaction of wheel running exercise and food restriction, even if the intake of other nutrients was low. Adequate energy intake also prevented estrous dysfunction. Our results provide evidence that adequate energy intake is important for optimal bone growth in young female athletes.

\section{Acknowledgements}

We thank MARUZEN-YUSHODO Co., Ltd. (https://kw.maruzen.co.jp/kousei-honyaku/) for the English language editing.

\section{Conflict of Interest}

The authors declare that they have no conflict of interest.

\section{References}

[1] Nattiv A, Loucks AB, Manore MM, Sanborn CF, Sundgot-Borgen J, Warren MP (2007) American College of Sports Medicine. American College of Sports Medicine position stand. The Female Athlete Triad. Med Sci Sports Exerc 39 (10): 1867-1882.

[2] Lauder TD, Dixit S, Pezzin LE, Williams MV, Campbell CS, Davis GD (2000) The relation between stress fractures and bone mineral density: evidence from active-duty Army women. Arch Phys Med Rehabil 81 (1): 73-79.

[3] Loucks AB, Kiens B, Wright HH (2011) Energy availability in athletes. J Sports Sci 29 Suppl 1: S7-S15.

[4] Talbott SM, Cifuentes M, Dunn MG, Shapses SA (2001) Energy restriction $\mathrm{r}$ educes bone density and biomechanical properties in aged female rats. J Nutr 131 (9): 2382-2387.

[5] Hawkins J, Cifuentes M, Pleshko NL, Ambia-Sobhan H, Shapses SA (2010) Energy Restriction Is Associated with Lower Bone Mineral Density of the Tibia and Femur in Lean but Not Obese Female Rats. J Nutr 140 (1): 31-37.

[6] Swift SN, Baek K, Swift JM, Bloomfield SA (2012) Restriction of dietary energy intake has a greater impact on bone integrity than does restriction of calcium in exercising female rats. J Nutr 142 (6): 1038-1045.
[7] Dimarco NM, Dart L, Sanborn CB (2007) Modified activity-stress paradigm in an animal model of the female athlete triad. J Appl Physiol 103 (5): 1469-1478.

[8] Aikawa Y, Agata U, Kakutani Y, Higano M, Hattori S, Ogata H, Ezawa I, Omi N (2015) The interaction of voluntary running exercise and food restriction induces low bone strength and low bone mineral density in young female rats. Calcif Tissue Int 97 (1): 90-99.

[9] Aikawa Y, Agata U, Kakutani Y, Kato S, Noma Y, Ogata H, Ezawa I, Omi N (2016) The preventive effect of calcium supplementation on weak bones caused by the interaction of exercise and food restriction in young female rats during the period from acquiring bone mass to maintaining bone mass. Calcif Tissue Int 98 (1): 94-103.

[10] Noda S, Sawaki M, Shiraishi K, Yamasaki K, Yamaguchi R (2002) Age-related changes of genital systems in the female Crj:CD (SD) IGS rats during sexual maturation. J Vet Med Sci 64 (4): 315-319.

[11] Anantharaman-Barr HG, Decombaz J (1989) The effect of wheel running and the estrous cycle on energy expenditure in female rats. Physiol Behav 46 (2): 259-263.

[12] Ezawa I, Okada R, Nozaki Y, Ogata, E (1979) Breaking-properties and ash contents of the femur of growing rat fed a low calcium diet. Nippon Eiyo Shokuryo Gakkaishi, J Jpn Soc Food Nutr 32 (5): 329-335.

[13] Omi N, Morikawa N, Ezawa I (1994) The effect of voluntary exercise on bone mineral density and skeletal muscles in the rat model at ovariectomized and sham stages. Bone miner 24 (3): 211-222.

[14] Omi N, Goseki M, Oida S, Sasaki S, Ezawa I (1994) The nutritional evaluation of globin on maintenance of bone metabolism in ovariectomized osteoporotic rats. J Nutr Sci Vitaminol 40 (5): 443-457.

[15] Cashman KD (2007) Diet, nutrition, and bone health. J Nutr 137 (11 Suppl): 2507S-2512S.

[16] Takeda S, Kobayashi Y, Park JH, Ezawa I, Omi N (2012) Effect of different intake levels of dietary protein and physical exercise on bone mineral density and bone strength in growing male rats. J Nutr Sci Vitaminol 58 (4): 240-246.

[17] Stubbs RJ, Hughes DA, Johnstone AM, Whybrow S, Horgan GW, King N, Blundell J (2004) Rate and extent of compensatory changes in energy intake and expenditure in response to altered exercise and diet composition in humans. Am J Physiol Regul Integr Comp Physiol 286 (2): R350-R358.

[18] Erben RG, Brunner KS, Breig B (2004) Long-term sensitivity of uterus and hypothalamus/pituitary axis to 17 beta-estradiol is higher than that of bone in rats. J Bone Miner 19 (11): $1827-1832$.

[19] Wang G (1923) The relation between "spontaneous" activity and oestrous cycle in the white rat. Comp Psychol Monogr 2: 1-27.

[20] Ammann P, Rizzoli R (2003) Bone strength and its determinants. Osteoporos Int 14 (Suppl 3): S13-S18.

[21] Omi N, Nakamura T, Ezawa I (1998) Modulation of bone mass and turnover in growing rats by voluntary weight-bearing exercise and glucose supplementation. J Nutr Sci Vitaminol 44 (3): 409-421. 
[22] Ackerman KE, Cano Sokoloff N, DE Nardo Maffazioli G, Clarke HM, Lee H, Misra M (2015) Fractures in Relation to Menstrual Status and Bone Parameters in Young Athletes. Med Sci Sports Exerc. 47 (8): 1577-86.

[23] Pando R, Masarwi M, Shtaif B, Idelevich A, Monsonego-Ornan E, Shahar R, Phillip M, Gat-Yablonski G (2014) Bone quality is affected by food restriction and by nutrition-induced catch-up growth. J Endocrinol 223 (3): 227-39.

[24] Cifuentes M, Morano AB, Chowdhury HA, Shapses SA (2002) Energy restriction reduces fractional calcium absorption in mature obese and lean rats. J Nutr 132 (9): 2660-2666.
[25] Shah BG, Trick KD, Belonje B (1990) Effects of dietary calcium on the metabolism of trace elements in male and female rats. J Nutr Biochem 1 (11): 585-591.

[26] Cashman KD, Flynn A (1996) Effect of dietary calcium intake and meal calcium content on calcium absorption in the rat. Br J Nutr 76 (3): 463-470.

[27] Ihle R, Loucks AB (2004) Dose-response relationships between energy availability and bone turnover in young exercising women. J Bone Miner Res 9 (8): 1231-1240. 